\title{
Efficacy of salt fluoridation
}

\author{
Abstracted from \\ Yengopal V, Chikte UM, Mickenautsch S, Oliveira LB, Bhayat A. \\ Salt fluoridation: a meta-analysis of its efficacy for caries prevention. SADJ 2010; 65: 60-67. \\ Address for correspondence: Dr V Yengopal, Division of Public Oral Health, \\ University of the Witwatersrand, 7 York Rd, Parktown, Johannesburg 2193, South Africa. \\ E-mail: veerasamy.yengopal@wits.ac.za
}

\section{Question: Is use of fluoridated salt an effective method of reducing caries in children's permanent teeth?}

Data sources Biomed Central, Cochrane Oral Health Group Trials Register, CENTRAL, Directory of Open Access Journals, Expanded Academic ASAP Plus, Metaregister of Controlled Trials, PubMed, ScienceDirect, Research Findings Electronic Register, BBO and LILACS. Study selection Studies reporting in English, Spanish or Portuguese were included if they reported on the caries preventive effect of salt fluoridation and provided mean DMFT scores with standard deviations or $95 \%$ confidence intervals. Randomised or quasi-randomised studies together with cross-sectional studies where historical control data were available for relevant cohorts were included. Studies were assessed for quality.

Data extraction and synthesis Data were extracted independently by two reviewers, with disagreements being resolved by discussion. Nine studies were included in a meta-analysis.

Results Two studies included 6-8 year-old children and showed a pooled reduction in DMFT scores of $-0.98(95 \% \mathrm{Cl}-1.68$ to -0.29$)$. The eight studies involving 9-12 year-old children showed a significant pooled DMFT reduction of $-2.13(95 \% \mathrm{Cl}-2.55$ to $-1.70, \mathrm{p}<0.0001)$, while the four studies with cohorts of 13-15 year-old children exhibited a great reduction in DMFT scores of $-4.22(95 \% \mathrm{Cl}-6.84$ to -1.59 , $\mathrm{p}<0.001)$. In one study that compared salt fluoridation with water fluoridation there was no statistical difference between the two groups. Conclusions The pooled estimates for each of the age cohort favoured salt fluoridation versus no exposure. However, due to the poor quality of the studies the contribution of fluoridated salt to the declines in DMFT could not be quantified. Thus, while this meta-analysis favours salt fluoridation, further high quality studies are needed to confirm its efficacy.

\section{Commentary}

Various political, geographical, financial and technical reasons have prevented the availability of water fluoridation to a large proportion of the world's population. The alternative approach of fluoridated salt was initiated in Switzerland in 1955. Successful community trials, World Health Organisation ${ }^{1}$ and FDI World Dental Federation recommendations; reconfirmation by the European Union of sodium and potassium fluoride as approved food additives; and adaption to local political, technological, and cultural environments, facilitated access to this approach for caries prevention. Salt fluoridation is identified by 12 countries in the Americas and 8 countries in Europe (estimate 250 million population), and several others have the product available. ${ }^{2}$

The aim of this meta-analysis was to assess the caries preventive effect of salt fluoridation in the permanent dentition in children. Although the inclusion and exclusion criteria were explicit, the reporting of this systematic review did not follow the recommendations from the PRISMA (Preferred Reporting Items for Systematic reviews and Meta-Analyses) Statement. ${ }^{3}$

The search strategy used to identify relevant studies cannot be considered as comprehensive because no controlled vocabulary was used in searching the electronic databases. During the literature search, two reviewers independently assessed the eligibility of abstracts. It is not clear whether study selection or data extraction was carried out independently or in duplicate.

Included studies were clearly laid out in table format and the level of evidence was rated using the hierarchy of evidence published in the fluoridation systematic review carried out by the Australian National Health and Medical Research Council (NHMRC). ${ }^{4}$ The inclusion criteria of these two systematic reviews are however different. This review has broadened the inclusion criteria to include studies which reported on cross-sectional caries status of specific age cohorts for whom historical controls could be obtained from other studies such as national oral health surveys. It should be noted that two comparative studies with concurrent controls ${ }^{5,6}$ were included in this systematic review; one of which ${ }^{5}$ had not been mentioned in the NHMRC review. The reason may be due to the fact that the study by Tóth ${ }^{5}$ was published in 1976, a year which was outside the search period (from 1996 onwards) of the NHMRC review. ${ }^{4}$ The other included study (with concurrent controls) ${ }^{6}$ was excluded from the NHMRC review ${ }^{4}$ because the cross-sectional data were not measured at multiple time-points.

Attempts were made to pool together data from included studies for different age cohorts by meta-analyses. The authors stated that there was significant statistical heterogeneity, which was related to the inconsistency in the size of the treatment effects and difference in sample sizes among the studies. It should also be noted that most included studies failed to provide information about demographic and other differences between the baseline and endpoint populations, and failed to adjust results for any differences and other potential contributing factors.

The authors of this review also conducted a systematic review of salt fluoridation in the primary dentition. ${ }^{7}$ Only two studies met 


\section{CARIES}

the inclusion criteria. It was concluded that there were significant caries preventive effects as measured by improvements in $\mathrm{dmft}$ or dft scores. These findings cannot, however, be considered as conclusive due to the limited number of included studies in the review. It should be noted that in this young age group, there is reason to believe that fluoridated salt does not give a caries preventive effect due to the low salt diet.

One concern expressed is that promotion of dental benefits of fluoridated salt would be unacceptable and contradictory to public health messages that encourage the reduction of consumption of salt and thus decrease the risk of hypertension. Populations of countries where fluoridated salt is available are not encouraged to consume more salt to improve their dental health; rather, the "automatic" or passive effect of fluoridated salt is accepted. In other words, people do not need to change their usual behaviour to benefit. Indeed, reduced consumption of salt could and should be encouraged and, where this is successful, the concentration of fluoride in salt could simply be increased appropriately. ${ }^{8}$

Salt fluoridation has the potential to reach entire populations but a substantial part of the population may be using non-fluoridated salt depending on local legislation concerning the availability and usage of fluoridated and non-fluoridated salt. In order to obtain substantial public health benefits, it has been hypothesised that at least $75-85 \%$ of the population need to use fluoridated salt. ${ }^{9}$ To assist individuals or groups of stakeholders in the selection or prioritisation of various fluoride interventions at the community, regional or national level, Yee and Holmgren have developed a Fluoride Intervention Template, a decision making matrix or tool, which is available on www.dentaid.org/resources/fluorideinter vantiontemplate.

\section{Practice point}

- There are insufficient studies with good quality evidence examining the effects of salt fluoridation in preventing dental caries. The included studies, however, suggested that fluoridated salt was beneficial to children, especially their permanent dentition.

C Albert Yeung

Department of Public Health, NHS Lanarkshire, Hamilton,

Scotland, UK

1. Peterson PE. World Health Organization global policy for improvement of oral health - World Health Assembly 2007. Int Dent J 2008; 58: 115-121.

2. Gillespie G. Historical development of salt fluoridation and its cariostatic effectiveness (abstract). OHDMBSC; 9: 186-187.

3. Moher D, Liberati A, Tetzlaff J, Altman DG, The PRISMA Group. Preferred Reporting Items for Systematic Reviews and Meta-Analyses: The PRISMA Statement. PLoS Med 2009; 6: e1000097.

4. National Health and Medical Research Council. A systematic review of the efficacy and safety of fluoridation. Canberra: National Health and Medical Research Council, 2007

5. Tóth K. A study of 8 year's domestic salt fluoridation for prevention of caries. Community Dent Oral Epidemiol 1976; 4: 106-110.

6. Fabien V, Obry-Musset AM, Hedelin G, Cahen PM. Caries prevalence and salt fluoridation among 9-year-old schoolchildren in Strasbourg, France. Community Dent Oral Epidemiol 1996; 24: 408-411.

7. Yengopal V, Chikte U, Mickenautsch S, Bhayat A. Caries preventive effect of salt fluoridation in the primary dentition (abstract). J Dent Res 2008; 87 (Spec Iss C): number 55 (S Afr Div).

8. Jones S, Burt BA, Petersen PE, Lennon MA. The effective use of fluorides in public health. Bull World Health Organ 2005; 83: 670-676.

9. Marthaler TM. Increasing the public health effectiveness of fluoridated salt. Schweiz Monatsschr Zahnmed 2005; 115: 785-792.

Evidence-Based Dentistry (2011) 12, 17-18. doi:10.1038/sj.ebd.6400776 